CMS RPC muon detector performance with 2010-2012 LHC data

This content has been downloaded from IOPscience. Please scroll down to see the full text. 2014 JINST 9 C12016

(http://iopscience.iop.org/1748-0221/9/12/C12016)

View the table of contents for this issue, or go to the journal homepage for more

Download details:

IP Address: 157.193.99.97

This content was downloaded on 17/06/2015 at $14: 56$

Please note that terms and conditions apply. 
$12^{\text {th }}$ Workshop on Resistive Plate Chambers and Related Detectors, 23-28 FEBRUARY 2014,

TSINGHUA UNIVERSITY, BEIJING, CHINA

\section{CMS RPC muon detector performance with 2010-2012 LHC data}

G. Pugliese, ${ }^{l, 1}$ Y. Ban, ${ }^{a}$ J. Cai, ${ }^{a}$ Q. Li, ${ }^{a}$ S. Liu, ${ }^{a}$ S. Qian, ${ }^{a}$ D. Wang,${ }^{a}$ Z. Xu, ${ }^{a}$ F. Zhang, ${ }^{a}$ Y. Choi, ${ }^{b}$ D. Kim, ${ }^{b}$ J. Goh, ${ }^{b}$ S. Choi, ${ }^{c}$ B. Hong, ${ }^{c}$ J.W. Kang, ${ }^{c}$ M. Kang, ${ }^{c}$ J.H. Kwon, ${ }^{c}$ K.S. Lee, ${ }^{c}$ S.K. Lee, ${ }^{c}$ S.K. Park, ${ }^{c}$ L.M. Pant, ${ }^{d}$ A.K. Mohanty, ${ }^{d}$ R. Chudasama, ${ }^{d}$ J.B. Singh, ${ }^{e}$ V. Bhatnagar, ${ }^{e}$ A. Mehta, ${ }^{e}$ R. Kumar, ${ }^{e}$ S. Cauwenbergh, ${ }^{f}$ S. Costantini, ${ }^{f}$ A. Cimmino, ${ }^{f}$ S. Crucy, ${ }^{f}$ A. Fagot,${ }^{f}$ G. Garcia, ${ }^{f}$ A. Ocampo, ${ }^{f}$ D. Poyraz, ${ }^{f}$ S. Salva,${ }^{f}$ F. Thyssen, ${ }^{f}$ M. Tytgat, ${ }^{f}$ N. Zaganidis, ${ }^{f}$ W. V. Doninck, ${ }^{g}$ A. Cabrera, ${ }^{h}$ L. Chaparro,${ }^{h}$ J.P. Gomez, ${ }^{h}$ B. Gomez, ${ }^{h}$ J.C. Sanabria, ${ }^{h}$ C. Avila, ${ }^{h}$ A. Ahmad, ${ }^{i}$ S. Muhammad,${ }^{i}$ M. Shoaib, ${ }^{i}$ H. Hoorani, ${ }^{i}$ I. Awan,,${ }^{i}$ I. Ali, ${ }^{i}$ W. Ahmed, ${ }^{i}$ M.I. Asghar, ${ }^{i}$ H. Shahzad,${ }^{i}$ A. Sayed, ${ }^{j}$ A. Ibrahim, ${ }^{j}$ S. Aly, ${ }^{j}$ Y. Assran,${ }^{j}$ A. Radi, ${ }^{j}$ T. Elkafrawy, ${ }^{j}$ A. Sharma,${ }^{k}$ S. Colafranceschi, ${ }^{k}$ M. Abbrescia, ${ }^{l}$ C. Calabria, ${ }^{l}$ A. Colaleo,,${ }^{l}$ G. laselli, ${ }^{l}$ F. Loddo, ${ }^{l}$ M. Maggi, ${ }^{l}$ S. Nuzzo, ${ }^{l}$ R. Radogna, ${ }^{l}$ R. Venditti, ${ }^{l}$ P. Verwillingen, ${ }^{l}$ L. Benussi, ${ }^{m}{ }^{m}$ S. Bianco, ${ }^{m}$ D. Piccolo, ${ }^{m}$ P. Paolucci, ${ }^{n}$ S. Buontempo, ${ }^{n}$ N. Cavallo, ${ }^{n}$ M. Merola, ${ }^{n}$ F. Fabozzi, ${ }^{n}$ O.M. Iorio, ${ }^{n}$ A. Braghieri, ${ }^{o}$ P. Montagna, ${ }^{o}$ C. Riccardi, ${ }^{o}$ P. Salvini, ${ }^{o}$ P. Vitulo, ${ }^{o}$ I. Vai, ${ }^{o}$ A. Magnani, ${ }^{o}$ A. Dimitrov, ${ }^{p}$ L. Litov, ${ }^{p}$ B. Pavlov, ${ }^{p}$ P. Petkov, ${ }^{p}$ A. Aleksandrov, ${ }^{q}$ V. Genchev,${ }^{q}$ P. laydjiev, ${ }^{q}$ M. Rodozov, ${ }^{q}$ G. Sultanov, ${ }^{q}$ M. Vutova, ${ }^{q}$ S. Stoykova, ${ }^{q}$ R. Hadjiiska, ${ }^{q}$ H.S. Ibargüen, ${ }^{r}$ M.I. P. Morales, ${ }^{r}$ S.C. Bernardino, ${ }^{r}$ I. Bagaturia, ${ }^{s}$ Z. Tsamalaidze, ${ }^{s}$ I. Crotty ${ }^{t}$ and M.S. Kim ${ }^{u}$ on behalf of the CMS collaboration

\footnotetext{
${ }^{a}$ Department of Technical Physics, Peking University, CN-100 871 Beijing, China

${ }^{b}$ Department of Physics, SungKyunKwan University,

2066, Seobu-ro, Jangahn-gu, Suwon, Gyeonggi-do, Republic of Korea

${ }^{c}$ Department of Physics and Korea Detector Laboratory, Korea University, Aman-dong 5-ga, Sungbuk-gu, Seoul, Republic of Korea

${ }^{d}$ BARC, Electronics Division (BARC), Trombay, Mumbai 400 085, India

${ }^{e}$ Department of Physics, Panjab University, Chandigarh Mandir 160 014, India
}

\footnotetext{
${ }^{1}$ Corresponding author.
} 
${ }^{f}$ Department of Physics and Astronomy, Ghent University, Proeftuinstraat 86, BE-9000 Ghent, Belgium ${ }^{g}$ VUB, Dienst Elementaire Deeltjes, Pleinlaan 2 BE-1050 Brussel, Belgium

${ }^{h}$ Universidad de Los Andes, Apartado Aéreo 4976, Carrera 1E, no. 18A 10, CO-Bogotá, Colombia

${ }^{i}$ Islamabad-NCP, Quaid-I-Azam University, Islamabad-44000 Pakistan

${ }^{j}$ Academy of Scientific Research and Technology of the Arab Republic of Egypt, 101 Sharia Kasr El-Ain, Cairo, Egypt

${ }^{k}$ CERN, CH-1211 Geneva 23, Switzerland

${ }^{l}$ Universita e INFN, Sezione di Bari, Via Orabona 4, IT-70126 Bari, Italy

${ }^{m}$ INFN, Laboratori Nazionali di Frascati, PO Box 13, Via Enrico Fermi 40, IT-00044 Frascati, Italy

${ }^{n}$ Universita e INFN, Sezione di Napoli,

Complesso Univ. Monte S. Angelo, Via Cintia, IT-80126 Napoli, Italy

${ }^{\circ}$ Universita e INFN, Sezione di Pavia, Via Bassi 6, IT-Pavia, Italy

${ }^{p}$ Atomic Physics Department, Faculty of Physics, University of Sofia,

5, James Bourchier Boulevard, BG-1164 Sofia, Bulgaria

${ }^{q}$ Inst. for Nucl. Res. and Nucl. Energy, Bulgarian Academy of Sciences,

Tzarigradsko shaussee Boulevard 72, BG-1784 Sofia, Bulgaria

${ }^{r}$ Benemérita Universidad Autónoma de Puebla, Av. San Claudio y 18 sur, edif. 111 A Ciudad Universitaria, Col. San Manuel, Puebla, Pue. C.P. 72570, Mexico

${ }^{s}$ Tibilisi-IHEPI, Tbilisi State University University Street 9 GE-380 086 Tbilisi, Georgia

${ }^{t}$ Department of Physics, University of Wisconsin, 1150 University Avenue Madison, WI 53706 U.S.A.

"Department of Physics, Kyungpook National University,

80 Daehakro, Bukgu, Daego, 702-701, Republic of Korea

E-mail: gabriella.pugliese@ba.infn.it

Abstract: The muon spectrometer of the CMS (Compact Muon Solenoid) experiment at the Large Hadron Collider (LHC) is equipped with a redundant system made of Resistive Plate Chambers (RPCs) and Drift Tube (DT) chambers in the barrel, RPC and Cathode Strip Chambers (CSCs) in the endcap region. In this paper, the operations and the performance of the RPC system during the first three years of LHC activity are presented. The stability of the RPC performance, in terms of efficiency, cluster size and noise, is also reported. Finally, the radiation background levels on the RPC system have been measured as a function of the LHC luminosity. Extrapolations to the High Luminosity LHC conditions are also discussed.

KEYWORDS: Resistive-plate chambers; Trigger detectors

ARXIV EPRINT: 1406.7543 


\section{Contents}

1 Introduction 1

2 Operation of the RPC system 2

3 RPC working point calibration 2

$4 \quad$ RPC performance stability 2

5 Noise and background rates $\quad 4$

6 Conclusion 6

\section{Introduction}

The Compact Muon Solenoid (CMS) is one of the four experiments at the LHC [1]. It is a multipurpose particle physics detector and is described in detail in [2]. The muon system is a relevant part of the apparatus. High momentum muons are produced in many Standard Model processes, like top quark, $\mathrm{W}$ and $\mathrm{Z}$ decay, and they are also an important signature of new physics channels, Higgs Boson decay on top of them [3]. Hence a robust and redundant muon spectrometer is needed to provide precise muon identification, good transverse momentum resolution measurement up to momenta of several $\mathrm{TeV}$, unambiguous charge identification and excellent trigger capability. The muon system, described in detail in [4], is the outermost detector of the CMS experiment, and it covers a pseudorapidity region $\eta<2.4$. The iron of the muon detector also acts as return joke for the 3.8 $\mathrm{T}$ solenoidal magnetic field produced by the superconducting inner magnet, thus keeping the size of the CMS detector relatively small, considering the field intensity. Three different gaseous detector technologies are employed: in the barrel, DT chambers detect muons up to pseudo-rapidity $|\eta|<1.2$; CSCs handle the higher rates and non-uniform magnetic field in the endcap region $0.9<|\eta|<2.4$; six and four planes of RPCs, in the barrel and the endcap region, respectively, cover the region of $|\eta|<1.6$. The barrel region is divided into 5 separate wheels (named $\pm 2, \pm 1$ and 0 ) while the endcaps in 4 disks both in the forward and backward directions (named $\pm 4, \pm 3$, $\pm 2, \pm 1$ ). The 4th disk has been installed during the LHC long shutdown in 2013-2014 (LS1). Each wheel is divided into 12 sectors while every disk into 36 sectors. In total there are 912 RPC chambers, covering an area of about $3500 \mathrm{~m}^{2}$, equipped with about 100.000 readout strips. The CMS RPCs are double-gap chambers with $2 \mathrm{~mm}$ gas width each and copper readout in between. The bakelite bulk resistivity is in the range of $2-5 \cdot 10^{10} \Omega \mathrm{cm}$. They operate in avalanche mode with a gas mixture of $95.2 \% \mathrm{C}_{2} \mathrm{H}_{2} \mathrm{~F}_{4}, 4.5 \% \mathrm{iC}_{4} \mathrm{H}_{10}$ and $0.3 \% \mathrm{SF}_{6}$ with $40 \%$ of humidity, working in closed loop mode with about $10 \%$ of fresh gas mixture. 


\section{Operation of the RPC system}

On March the 30th 2010, LHC started proton-proton collisions at a center-of-mass energy of $7 \mathrm{TeV}$. The year long machine commissioning has turned into an increase of the maximum instantaneous luminosity, achieving up to $2 \cdot 10^{32} \mathrm{~cm}^{-2} \mathrm{~s}^{-1}$ by the end of 2010. In 2011, there was enormous increase in luminosity, such that an integrated luminosity equal to that of the entire dataset of 2010 was delivered in less than a day. The instantaneous luminosity reached up to about $3.5 \cdot 10^{33} \mathrm{~cm}^{-2} \mathrm{~s}^{-1}$, setting a new world record for beam intensity at a hadron collider. In 2012, LHC has been operating at the increased energy of $4 \mathrm{TeV}$ per beam, reaching a maximum of instantaneous luminosity of about $6.6 \cdot 10^{33} \mathrm{~cm}^{-2} \mathrm{~s}^{-1}$. In the period 2010-2012, called RUN1, the total delivered luminosity was $30 \mathrm{fb}^{-1}$, out of this $27 \mathrm{fb}^{-1}$ was recorded by CMS. The results discussed in the following sections are based on data collected during RUN1.

The RPC system performed extremely well: the contribution to the CMS downtime was below $1.5 \%$, quite constant every year. The fraction of active channels was between $97.5 \%$ and $98 \%$ for the whole length of RUN1. The fraction of dead (masked or inactive) channels was mainly caused by noisy chambers due to faulty electronic boards located inside the chambers (not accessible since 2009) or failures of HV/LV channels. Most of the dead channels have been already recovered during LS1 [5].

\section{RPC working point calibration}

During 2010, the LHC low luminosity did not allow to fine-tune the Hight Voltage (HV) settings chamber by chamber. Only two different values were chosen to operate the endcap and barrel chambers $(9.5 \mathrm{kV}$ and $9.3 \mathrm{kV}$, respectively). These values were defined on the basis of measurements performed during the construction and commissioning phase. Once in early 2011 and twice in 2012, HV calibrations were carried out on collision data to optimize the chamber Working Point (WP) and monitor as a function of the time the performance. The chamber WP has been defined as the $\mathrm{HV}$ corresponding to the $95 \%$ of the maximum efficiency plus $100 \mathrm{~V}$ for the barrel and $150 \mathrm{~V}$ for the endcap. Muon events have been selected, thanks to the redundancy of the muon system, asking for a DT or CSC trigger. Then a linear extrapolation of the track segment in the DT and CSC chambers was performed toward the closest RPC strip plane and then matched to any RPC cluster in a range of 8 strips around the extrapolated impact point. More details on the method can be found in [6]. In order to take into account atmospheric pressure variations, the applied HV was corrected for pressure variations defining the effective $\mathrm{HV}\left(\mathrm{HV}_{\text {eff }}\right)$, by the well-known formula [7]. As the temperature of the experimental hall was overall constant in time, no temperature correction was applied. Figure 1 shows the average efficiency for all the barrel and endcap chambers as a function of $\mathrm{HV}_{\text {eff }}$ and the $\mathrm{HV}_{\text {eff }}$ distributions as measured at $50 \%$ of the efficiency, in 2011 and 2012. No significative variations have been observed on both working point and chamber efficiency plateau values. The high voltage shift between barrel and endcap chambers depends on few microns difference in the spacers width (located inside the gas gap).

\section{RPC performance stability}

During RUN1, the chamber performance was measured run-by-run allowing to monitor the stability of the system. In figure 2 the barrel average cluster size and efficiency as a function of time are 

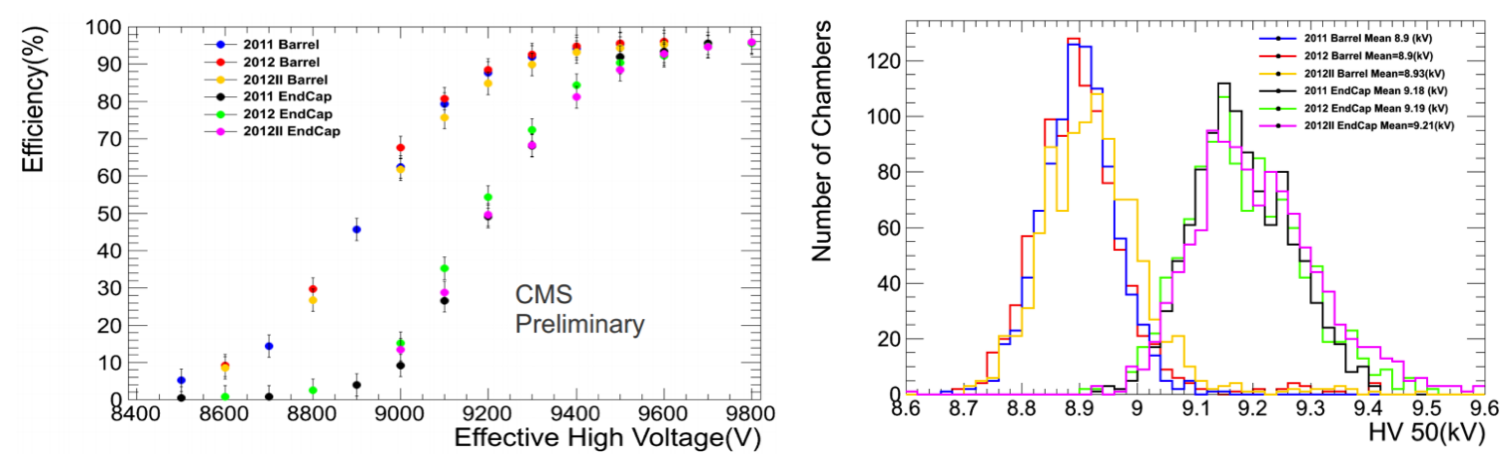

Figure 1. Left: barrel and endcap average efficiency as a function of the $\mathrm{HV}_{\text {eff }}$ as measured with the $\mathrm{HV}$ calibrations in 2011 and 2012. Right: the $\mathrm{HV}_{\text {eff }}$ distributions as measured at the $50 \%$ of the efficiency in the same runs.
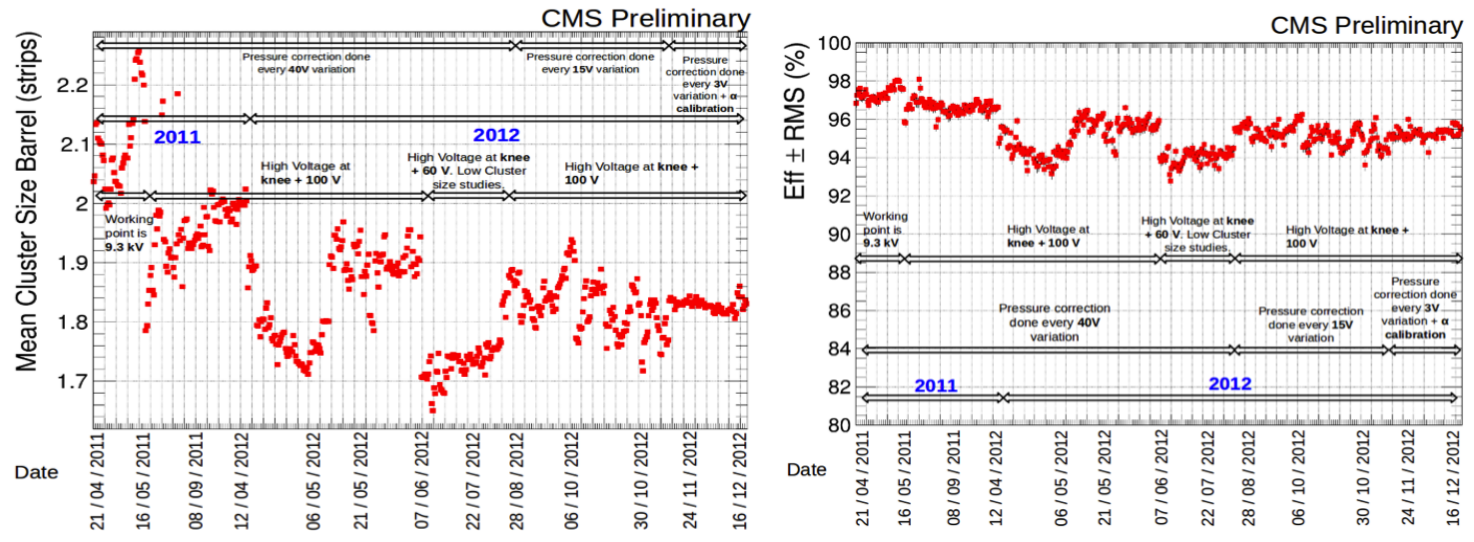

Figure 2. Left: barrel cluster size vs. time. Right: barrel average efficiency vs. time. The horizontal arrows separate runs taken applying different procedure for HV pressure correction.

shown. The different running periods, also marked by the horizontal arrows and highlighted in the plots, correspond to different algorithms used to correct the applied HV for the variations of the atmospheric pressure. At beginning, the WPs were corrected only when pressure variation translated into a variation of the $\mathrm{HV}$ of $40 \mathrm{~V}$; later, at the end of July 2012, this value was reduced to $15 \mathrm{~V}$ and finally, in November 2012, to $3 \mathrm{~V}$. Moreover, it was observed that the HV pressure correction formula slightly over-correct the effects due to pressure [8]. Therefore, during the last period of running the formula was modified with the following one, reducing $\alpha$ from 1 to 0.8 (where $p_{0}=965$ mbar):

$$
H V=H V_{\mathrm{eff}}\left(1-\alpha+\alpha p / p_{0}\right)
$$

As it can be inferred from the figure 2, the improvement on performance stability is evident: in particular the fluctuations of the average efficiency have been reduced from $\pm 3 \%$ to $\pm 0.5 \%$, at the end of running. Keeping performance stable over the time was one of the greatest successes achieved during the 2012 data taking period.

Figure 3 shows the chambers efficiency map as measured in 2012 for one wheel and for one disk. Most of the chambers have an efficiency of more than 95\%, except for few cases, whose lower efficiency is due to known hardware problems. 

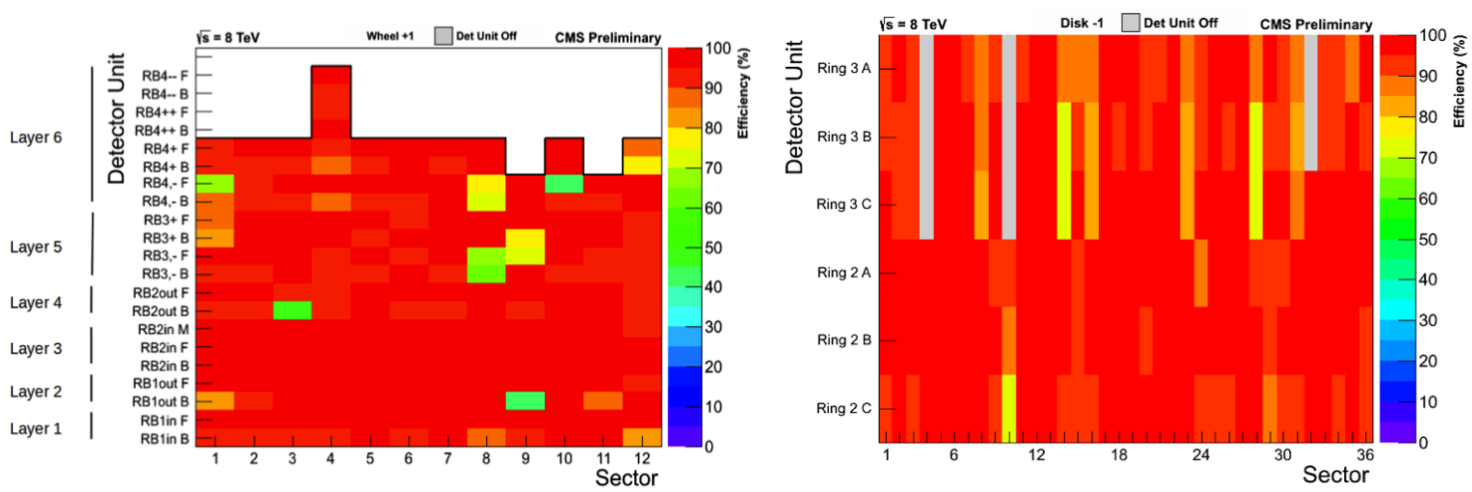

Figure 3. Left: chambers efficiency map for the wheel +1 . The plot shows the 12 sectors on $x$ axis and the 6 RPC layers on $y$ axis. Right: chambers efficiency map in disk -1. The 36 sectors are shown on $x$ axis and the 3 layers on $y$ axis.
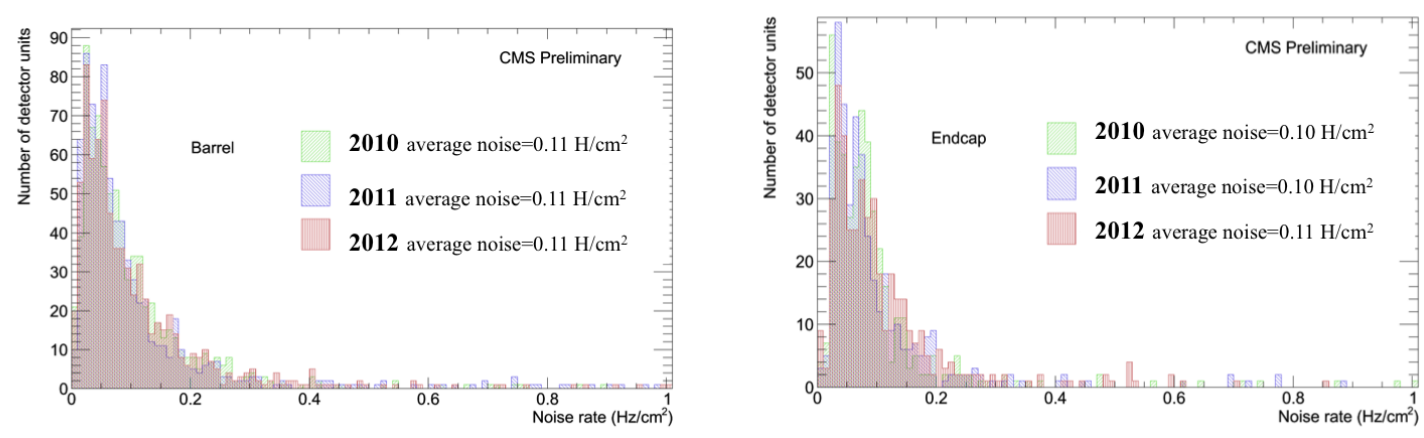

Figure 4. Distributions of the noise rate for barrel (left) and for the endcap (right) for the 3 years running 2010-2012.

\section{Noise and background rates}

The intrinsic chambers noise and background radiation levels can have an important impact on the overall performance of the system: high values might affect the trigger performance and the pattern recognition efficiency of muon tracks. Before every proton beam fill, the intrinsic noise rate is measured in order to mask noisy strips where needed. Figure 4 shows the intrinsic noise distributions for barrel and endcap chambers. The average values, of about $0.1 \mathrm{~Hz} / \mathrm{cm}^{2}$, are well below the CMS requirements and stable during the whole running period.

The background radiation in the muon chambers arises from low-energy gamma rays and neutrons from p-p collisions, low-momentum primary and secondary muons, punch-through hadrons from the calorimeters, muons and other particles produced in the interaction of the beams with collimators, residual gas and beam pipe elements. Figure 5 shows the average background rates versus the instantaneous luminosity in 2012 for each of the five wheels. As expected, the rates increase as the chambers are farther from the IP: wheel 0 is the closest one to the interaction point, +2 and -2 are the farthest. 


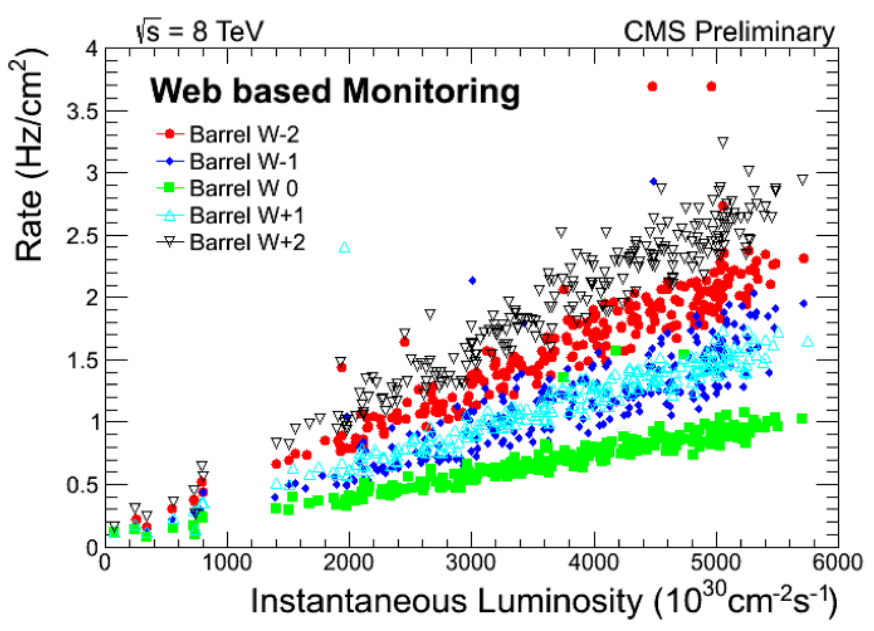

Figure 5. Measured background rate in the wheels as a function of the LHC luminosity in 2012 (wheel 0 is the closest one to the interaction point, +2 and -2 are the farthest).
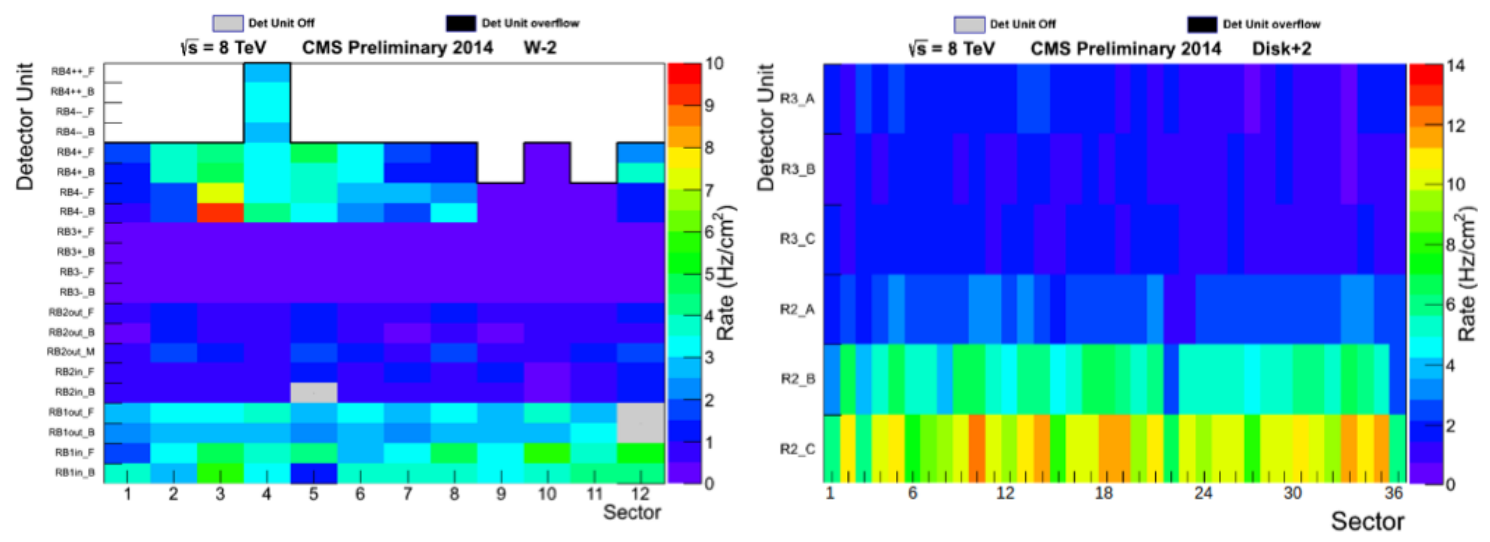

Figure 6. Left: measured noise rate map in one of the barrel wheel -2. Right: measured noise rate map in one of the endcap disk +2 . LHC luminosity about $4 \cdot 10^{33} \mathrm{~cm}^{-2} \mathrm{~s}^{-1}$.

Figure 6 shows the chambers rate map as measured in one barrel wheel and one endcap disk at a luminosity of $4 \cdot 10^{33} \mathrm{~cm}^{-2} \mathrm{~s}^{-1}$. In the barrel region, the highest rates $\left(\sim 7 \mathrm{~Hz} / \mathrm{cm}^{2}\right)$ are in the outermost stations (RB4), mainly due to diffused neutron background, and in the innermost stations (RB1), to charged particles and punch trough hadrons. In the endcap region the highest rate $\left(\sim 12 \mathrm{~Hz} / \mathrm{cm}^{2}\right)$ is measured in the disk 2 inner ring. More details can be found [9].

At LHC and at the High Luminosity phase of the LHC (HL-LHC), a center-of-mass energy of $14 \mathrm{TeV}$ and peak luminosity of $2 \cdot 10^{34} \mathrm{~cm}^{-2} \mathrm{~s}^{-1}$ and $5 \cdot 10^{34} \mathrm{~cm}^{-2} \mathrm{~s}^{-1}$, respectively, are expected. Table 1 summarizes the expected maximum and average background rates obtained by extrapolating linearly the RUN1 data. Such high radiation background will push the RPC system beyond its original lifetime [10, 11], requiring a new assessment of the detector longevity for HL-LHC. 
Table 1. Expected maximum and average background conditions at LHC luminosity of $2 \cdot 10^{34} \mathrm{~cm}^{-2} \mathrm{~s}^{-1}$ and at HL-LHC luminosity of $5 \cdot 10^{34} \mathrm{~cm}^{-2} \mathrm{~s}^{-1}$. No safety factor is included in the estimation.

\begin{tabular}{|c|cc|cc|}
\hline & \multicolumn{2}{|c|}{$\mathrm{L}=2 \cdot 10^{34} \mathrm{~cm}^{-2} \mathrm{~s}^{-1}$} & \multicolumn{2}{c|}{$\mathrm{L}=5 \cdot 10^{34} \mathrm{~cm}^{-2} \mathrm{~s}^{-1}$} \\
& Max. rate $\left(\mathrm{Hz} / \mathrm{cm}^{2}\right)$ & $<$ Rate $>\left(\mathrm{Hz} / \mathrm{cm}^{2}\right)$ & Max. rate $\left(\mathrm{Hz} / \mathrm{cm}^{2}\right)$ & $<$ Rate $>\left(\mathrm{Hz} / \mathrm{cm}^{2}\right)$ \\
\hline Barrel & 50 & 10 & 125 & 25 \\
Endcap & 100 & 25 & 250 & 60 \\
\hline
\end{tabular}

\section{Conclusion}

The operation of the CMS RPC muon system during the first three years of LHC data taking was outstanding: the contribution to CMS downtime was below $1.5 \%$ and the fraction of active channels was about $97.5 \%$ at the end of RUN1. Most of the inactive channels have been already recovered during LS1. After 3 years of LHC running with increasing instantaneous luminosity and 6 years from the end of RPC construction, the detector performance is within the CMS specifications and stable with no degradation observed. The monitoring as a function of the time of the RPC performance shows an average efficiency above $95 \%$, with variations below $\pm 0.5 \%$ at the end of RUN1. The expected background rate at a luminosity of $5 \cdot 10^{34} \mathrm{~cm}^{-2} \mathrm{~s}^{-1}$ will be sustainable for the present RPC system, although new aging tests are needed to certify the system for 10 years of HL-LHC activity.

\section{References}

[1] L. Evans and P. Bryant, LHC machine, 2008 JINST 3 S08001.

[2] CMS collaboration, The CMS experiment at the CERN LHC, 2008 JINST 3 S08004.

[3] CMS collaboration, Combined results of searches for the standard model Higgs boson in pp collisions at $\sqrt{s}=7 \mathrm{TeV}$, Phys. Lett. B 710 (2012) 26.

[4] CMS collaboration, The Muon Project Technical Design Report, CERN-LHCC-97-32 (1997).

[5] CMS collaboration, CMS RPC Commissioning of the Existing Detector During the Long Shutdown, in these proceedings.

[6] CMS collaboration, The performance of the CMS muon detector in proton-proton collisions at $\sqrt{s}=7 \mathrm{TeV}$ at the LHC, 2013 JINST 8 P11002.

[7] M. Abbrescia et al., Resistive plate chambers performances at cosmic rays fluxes, Nucl. Instrum. Meth. A 359 (1995) 603.

[8] S. Costantini et al., Uniformity and stability of the CMS RPC detector at the LHC, 2013 JINST 8 P03017.

[9] CMS collaboration, RPC Radiation background at the CMS experiment, in these proceedings.

[10] H.C. Kim et al., Quantitative aging study with intense irradiation tests for the CMS forward RPCs, Nucl. Instrum. Meth. A 602 (2009) 771.

[11] M. Abbrescia et al., Study of long-term performance of CMS RPC under irradiation at the CERN GIF, Nucl. Instrum. Meth. A 533 (2004) 102. 